\title{
ENERGY EFFICIENT DATA COLLECTION SCHEME FROM CHAINED-CLUSTER HEADS IN LARGE SCALE MSNs
}

\author{
Suma $\mathrm{G}^{\# 1}$, M.Siddappa ${ }^{* 2}$ \\ ${ }^{\# 1}$ Research Scholar, Sri Siddhartha Academy of Higher Education [SAHE],Tumkur, India. \\ 1 sumagkote@gmail.com \\ ${ }^{*}$ Dept. of Computer Science \&Egg.Sri Siddhartha Institute of Technology,Tumkur, India. \\ 22siddappa.p@gmail.com
}

Abstract-In present scenario Mobile Sensor Networks (MSNs) have gained lot of attention as a benefiting technology in many applications. MSN with static sink suffers from uneven energy consumption of nodes. Mobile Sink (MS) has expunged this drawback to a certain extent. But data collection through MS introduces more latency. Therefore, swift and efficient data collection is a challenge in large MSNs. This work attempts to reduce communication distance by forming multiple chains of $\mathrm{CHs}$ present in a particular region.MS collects accumulated data from Chain-Cluster Head (C$\mathrm{CH})$.Performance is evaluated through simulation and found improvement in terms of network lifetime, reduced energy consumption and data collection latency.

Keyword-Chain clusters, Mobile collector, Energy efficiency, latency.

\section{INTRODUCTION}

Developments in technology has led to productionof low power tiny sensors. These are deployed in large numbers in sensing area to monitor or to track continuously and report data to sink. Generally, nodes are static in wireless sensor networks (WSNs), but mobile nodes are used in mobile sensor networks to monitor harsh environment or improve performance in terms of life time, coverage and latency. Battery of sensors is limited and not easily replaceable in sensitivity application as they demand continuous monitoring. It is expected that algorithms to be energy efficient. Extending battery life by optimizing its usage is one of MSNs challenge. Energy balancing among sensors is very essential to improve network life[1][2].Clustering in homogenous sensor network reduces energy consumption and increases its life. Motes with high residual energy at beginning of rounds are selected to lead clusters[3][4] .If these cluster head(CH) lead clusters for a long time their energy depletes fast and dies early .Dead nodes hamper communication path and network spends more energy to forward data to sink, deteriorates MSNs life time[5].LEACH is a popular cluster based protocol ,energy threshold is used to rotate $\mathrm{CH}$ roles. $\mathrm{CH}$ communicate directly with outside sink ,this causes major energy draining in $\mathrm{CH}$. Researchers have proposed various techniques to reduce nodes battery usage, such as mobile sink, data aggregation , multi path routing and cluster chaining .

Connected clusters reduce CHs' communication distance to sink in turn reduces energy consumed for communicating to sink [6]. Nodes communicate only with neighbours present within communication range. Chain leaders are selected and are responsible for data forwarding to sink placed outside sensing field. These nodes relive nodes from communicating directly with sink and causing less depletion of energy. Further mobile sink improves nodes energy conservation but proper planning of its movement in sensor field is essential. Sinks' movement information updates influence routing algorithm performance such as data delivery ratio and delay. A mobile sink reduces sensor energy consumption spent on forwarding data to sink and also formation of dead nodes near sink. If mobile sinks' arrival time known to nodes in advance, their duty cycles may be adjusted and bring them out of sleep-mode on sinks' arrival [3]. This reduces nodes' energy usage in its active mode. Both techniques are energy conserving techniques and MS collects data directly with minimum latency. This work is a culmination of cluster chaining and data collection by MS from the C-CH node and a comparative analysis of proposed work with "MERA"[8], is carried out.

Motivation: Routing the sensed data to sink consumes remarkable amount of nodes' energy. Clustering reduces energy consumption at intra cluster level. If cluster coverage area is small, then data travels for small distance within clusters. Inter cluster communication by multi hop path distance is more if each $\mathrm{CH}$ is allowed to transmit data to sink independently. Hence nodes taking part in this communication lose more energy than other nodes and die fast. There by reducing networks overall life time. In order to reduce inter cluster communication distance, cluster heads are linked and data of more than one cluster is collected at one node, from this node MS collects data during its visit. Chained CHs reduces nodes energy consumption but increases 
data delivery delay. Further to decrease data delivery delay mobile sink is used for data collection. A combination of chained $\mathrm{CHs}$ and mobile sink optimizes energy consumption and data communication latency in a dynamic topology.

Organization of the paper: Rest of the paper is organized as follows: In section II some cluster linking and mobile sink related algorithms are reviewed. Section III proposes an energy efficient data collection scheme from chained-cluster heads in large scale MSN is discussed. Section IV consists of results discussions obtained through simulation of chained CHs along with MS. The work is concluded and future research scope is presented in section $\mathrm{V}$.

\section{RELATED WORK}

Some of earlier works in the field of linking nodes in clusters or linking $\mathrm{CH}$ are presented in first section. In second part works on mobile sink for data collection is presented.

\section{A. Connected Clusters}

In PEGASIS protocol [7] nodes communicate only with neighbours within communication range forming chains. Chain leaders are selected and are responsible for data forwarding to sink placed outside sensing field. These nodes relive nodes from communicating directly with sink and causing less depletion of energy. Samaleswari et al.,[8]presents "MERA" an energy efficient multi chained protocol, neighbouring nodes are connected to one another and chain clusters are formed and one chain leader is selected. In next stage neighbouring cluster chain heads are linked and aggregated data is transmitted to static sink situated outside sensor field. Feilong Tang et al., in [9], have divided the sensor area into chain areas. Nodes in these areas form a chain and transmit sensed data to $\mathrm{CH}$. Further a cluster is formed only considering chain head nodes for inter cluster communication to sink. Dawei et al., in [10] cluster linking is proposed to overcome unequal energy consumption of nodes energy. The process of chaining starts from low residual energy node within cluster and proceeds in ascending order of nodes' residual energy until all nodes are covered in one cluster. As nodes within a cluster are linked this works on local information and minimum overheads. This balances the nodes energy by starting the chain from least energy node.

\section{B. Mobile sink.}

Mariam Alnuaimiet al.,[11]presents a data collection algorithm in large delay tolerant networks using mobile sink multi hop path reduces energy by nodes but increases delivery time i.e latency. Algorithm is implemented in virtual grids; grid size is the sensor range. Each grid has a check point, to which ferry visits and collects data. Ferry collects data from all CHs and after collecting from all CH delivers to sink. Ming Ma et al.,[12] proposes multiple collectors are usage to collect data from nodes directly present within single hop distance. As data is collected directly from sensors no aggregation process, no relay nodes are used and data collision is absolutely reduced to zero. In this work more, importance is given to tour planning of mobile collectors in order to reduce their tour length and latency. Miao Zhao

etal.,[13]presents a mobile collector to collect data from CH.MC is equipped with two antennas to collect data from two $\mathrm{CH}$ simultaneously. Hence MC selects two suitable $\mathrm{CHs}$ and visits them at a common point between them and collects data from them at the same time. The data collection time is remarkably reduced. In work [14] data of every cluster is collected at collection points. Every cluster has one collection point. Among these collection points a collection tree is constructed iteratively till a optimal path is obtained. The tour length is maintained below a fixed value for any given application. It is understood that linking of clusters converses nodes energy and mobile collector reduces data collection latency and elongates networks lifetime.

\section{PROPOSED WORK}

In this section, the working principle of the proposed energy efficient data collection scheme from chainedcluster heads in large scale MSN is discussed. And various phases of the proposed work areanalysed in detail.

Working:

The main objective of this work is to optimize energy consumption and delay and improve residual energy. Main working phases of the algorithm are,

1. Deployment, clustering and reclustering

2. Formation of chains.

3. Data communication.

Deployment, clustering and reclustering: Initially sensor field is divided into ' $n$ ' first level virtual grids and further these grids are divided into 'n' second level virtual grids. In these second level grids CHs are selected and clusters are formed as in Figure 1. Cluster formation and $\mathrm{CH}$ selection is performed by sink. $\mathrm{CH}$ selection is based on nodes remaining energy, density, location and mobility (speed) of node. Once the clusters are formed they are maintained until either their structure changes or a $\mathrm{CH}$ rotation condition arises. Global and local hybrid reclustering is used similar to [15]. 


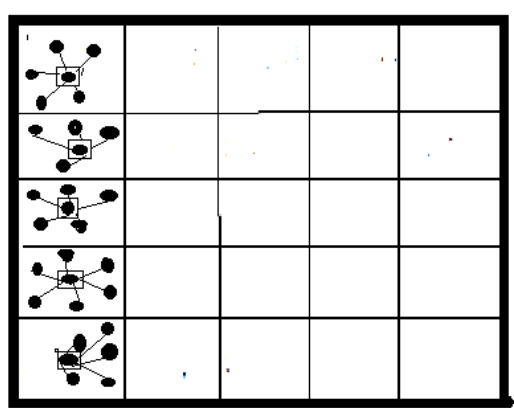

Figure1.Clusters.

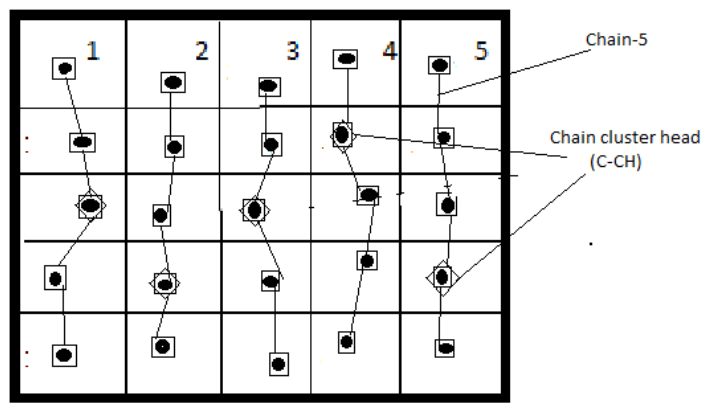

Figure 2. Chains

Chain-formation: The CHs of second level clusters present within designated first level grids are linked to one another to form a chain. In this way all CHs present in first level grids are

linked to one another to form multiple chains, Figure 2. Number of chains is equal to number of first level virtual grids. Among the linked $\mathrm{CHs}$ one $\mathrm{CH}$ is selected to act as data access point for mobile sink. This is called as Chain-Cluster Head(C-CH) and has high residual energy when compared to other $\mathrm{CHs}$ present in the chain. Nodes play the role of member,CH or C-CH depending ontheir residual energy, location and density.

Data communication: Data communication from sensor nodes to sink is known as communication phase. This takes place in following phases,

- $\quad$ Sensors to $\mathrm{CH}$ communication,

- $\quad \mathrm{CH}$ to $\mathrm{C}-\mathrm{CH}$ communication

- $\quad$ C-CH to sink communication

In hierarchical routing is usually performed in two separate phases as intra and inter cluster communication. In this work data routing is carried out in three phases, due to the formation of $\mathrm{CH}$ chains.

Sensor to $\mathrm{CH}$ communication is multi hop, if source node is unable to find a neighbour node it transmits directly to $\mathrm{CH}$. In this work an additional phase is introduced i.e $\mathrm{CH}$ to $\mathrm{C}-\mathrm{CH}$ communication .This communication is multi hop and in absence of neighbours, forwarding CHdirectly transmit to C-CH. In this way cluster heads are relived from transmitting to sink, as they forward their data to next $\mathrm{CH}$ and finally to $\mathrm{C}-\mathrm{CH}$ in their chain. In this way all linked CHs forward their data to C-CH in high level cluster, Figure 3.
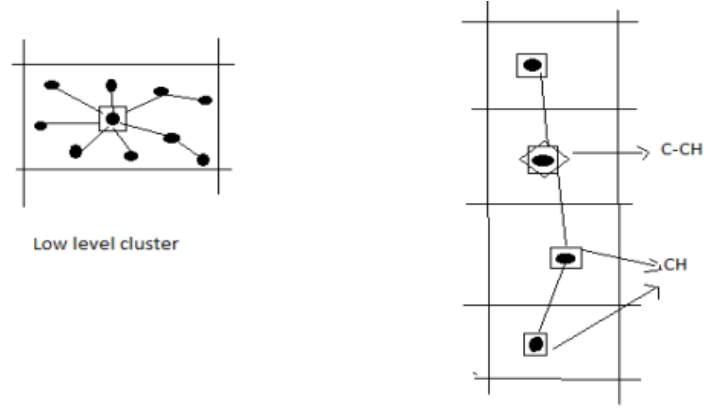

High level cluster

Figure 3. Cluster Hierarchy

C-CH to sink: CHs of low level clusters present in one first level grid (level-1) are connected to one another to form a chain. Therefore, number of chains is equal to number of regions formed in level-1. Sink selects one $\mathrm{CH}$ with highest residual energy among the chained $\mathrm{CHs}$ and elevates it to next level to further forward the accumulated data of all clusters present in that chain to sink. As this node acts as head node for the respective chain it is called as Chain-CH.

For evaluation purpose three cases of sink placement is considered.

i) Static sink placed outside,the data collected at $\mathrm{C}-\mathrm{CH}$ is transmitted through multi hop routes comprising of en route CHs and nodes within communication range of transmitting node. In the absence of neighbours,the transmitting node is allowed to transmit directly to sink without wasting time to attain minimumlatency. By Figure 3, the residual energyperformance of OBS is better than [15] withmulti hop routing without chain but has increased delay. 
ii) Staticsink placedinside; to reduce the multihoppath distance static sink is placed at the centre of sensor field.The communication from C-CH to sink is similar to outside sink scenario. As seen in Figure 6, due to reduced path length energy consumption and delay is less as compared to outside static sink [8].

iii) Mobile sink for data collection from $\mathrm{C}-\mathrm{CH}$,tofurther reduce the data collection delay and energy consumption, MS is used to collect data from C-CH directly. This reduces the path length for which data has to travel to sink to zero.The location of C-CH is known to sink hence the tour planning of MS is performed using Travelling Sales Man Problem (TSP). In which MS visits all C-CHs only once for data collection traversing in shortest path and returns back to its initial position. As all sensors are location aware the path on which MS traverses is pre-definedfor every data collection round, hence requires no announcement of sinks' arrival.

\section{Scheduling for reduced energy consumption}

Energy consumed by nodes for transmitting is $\mathrm{E}_{\mathrm{tx}}$,

$E_{t x}=\left(E_{\text {ele }} * k+E_{\text {amp }} * k\right) d^{2}$

Where, $\mathrm{E}_{\mathrm{tx}}$ is the Energy spent for transmitting data/byte/meter,

$\mathrm{E}_{\text {ele }}$ energy consumed by receiver or transmitter circuitry,

$E_{\text {amp }}$ energy spent at transmitter amplifier,

$\mathrm{k}$ is number of bits of data transmitted,

$\mathrm{d}$ is the distance between transmitting and receiving node.

Energy spent by nodes to receive ' $\mathrm{k}$ ' bits is $\mathrm{E}_{\mathrm{rx}}$,

$E_{r x}=\left(E_{\text {ele }} * k\right)$

\section{Energy spent by nodes in chain,}

Energy spent by first chain members, present at both ends of chain is,

$E_{c m 1}=E_{t x}+E_{m}$

Where, $E_{m}$ is the Energy spent formobility.

First chain members only transmit data to next node in chain.

Energy spent by other chain member nodes,

$E_{c m}=\left(E_{t x}+E_{r x}\right)+E_{m}$

These members transmit to next chain member and receive from the previous chain member only.

Total Energy spent by chain member nodes is,

$T E_{c m}=\left(\left(E_{c m} *(m-3)\right)+E_{c m 1} * 2\right)$

Where, $\mathrm{m}=$ number of $\mathrm{CHs}$ present in the chain.

$\mathrm{C}-\mathrm{CH}$ receives data from only two adjacent nodes present in chain and (m-3) nodes are chain members excluding $\mathrm{C}-\mathrm{CH}$ and two members $\mathrm{CH}$ present at both ends of the chain.

Energy spent by C-CH node is,

$E_{C-C H}=\left(\left(E_{\text {ele }} * k+E_{\text {amp }} * k\right) d^{2}+\left(E_{\text {ele }} * k\right) 2\right)+E_{m}$

Substituting from Eq (1)(2)

$E_{C-C H}=E_{t x}+E_{r x}+E_{m}$

Total energy spent by nodes in one high level cluster (chain), Eq (5) + (7)

$\boldsymbol{E} \boldsymbol{T}_{\text {chain }}=\boldsymbol{T} \boldsymbol{E}_{\text {cm }}+\boldsymbol{E}_{\boldsymbol{C}-\boldsymbol{C H}}{ }^{(8)}$

Table 1. Details of Simulation Settings

\begin{tabular}{|l|c|}
\hline \multicolumn{1}{|c|}{ Parameters } & \multicolumn{1}{c|}{ Values } \\
\hline Network area & $500 \times 500$ \\
\hline Number of nodes & 199 \\
\hline Sink & 1 \\
\hline Nodes mobility & $10 \mathrm{mts} / \mathrm{mint}(\mathrm{max})$ \\
\hline One simulation time & 't' \\
\hline
\end{tabular}




\section{RESULTS AND DISCUSSIONS:}

Network Model:

Assumptions made are as follows,

- $\quad \mathrm{N}$ sensor nodes are deployed in an M x M square field.

- All sensor nodes are homogenous -similar configuration in terms of sensing and Communication range.

- $\quad$ Sensors are location aware, possess unique ID and mobile.

- Nodes use power control to adjust the amount of transmitting power.

- Nodes death is not accidental, but only due to reduced battery level below set threshold it dies and is not allowed to participate in further activities.

Definition 1 :( Neighbouring nodes), Nodes within the communication range of transmitting node are considered as neighbouring nodes.

Definition 2 :( Large scale network), Network in which deployed nodes have no direct communication with BS.

Definition 3 :( One simulation time-' $t$ '), Time Period between nodes sensing and data received by sink.

Clusters are formed in grids. A combination of local and global reclusteringis used to recluster and for $\mathrm{CH}$ rotation. A node with highest weight is selected as $\mathrm{CH}$. Residual energy, mobility and density are the parameters used for selection. Inter cluster communication used is multi hop [15]. Node deployment and network settings are as listed in table 1.

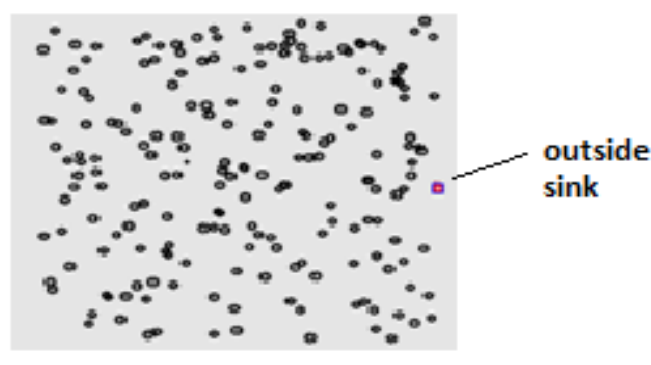

Figure 4. Sensor Deployment with OBS

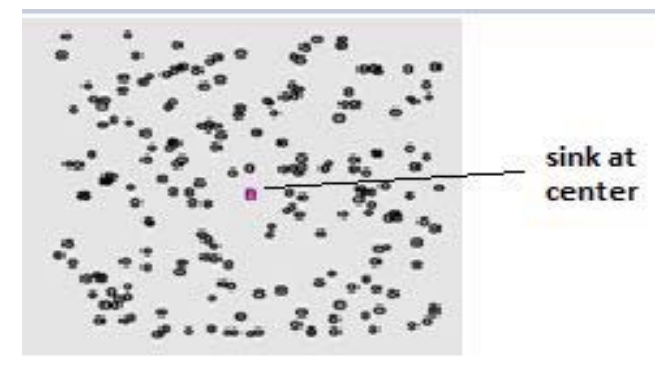

Figure 5. SensorDeployment with CBS

Simulation Scenario:

To determine the best sink placement in the sensor field andanalysethe impact of sink mobility on reducing data collection latency the algorithm is evaluated for following combinations.

Case 1-Static sink: i) placed outside sensor field (OBS), Figure 4.

ii) Placed inside at centre of sensorfield (CBS), Figure 5.

Case 2-Mobile sink initial location is at centre.

Forthese cases clustering Conditions are similar to [15]. Further CHs are chained and C-CH is selected and used for further routing to sink. The cases differ from one another only in data routing or collection technique from $\mathrm{C}-\mathrm{CH}$ to sink.

TABLE 2. Residual Energy

\begin{tabular}{|l|c|c|c|c|}
\hline & \multirow{2}{*}{$\begin{array}{l}\text { MULTI HOP } \\
\text { (without chain) [ 15] }\end{array}$} & \multicolumn{3}{|c|}{ WITH CHAIN } \\
\cline { 3 - 5 } & & Static OBS & Static OBS & MBS \\
\hline RESUIDAL-ENERGY & 97.6785 & 98.8097 & 98.8167 & 98.8223 \\
\hline
\end{tabular}




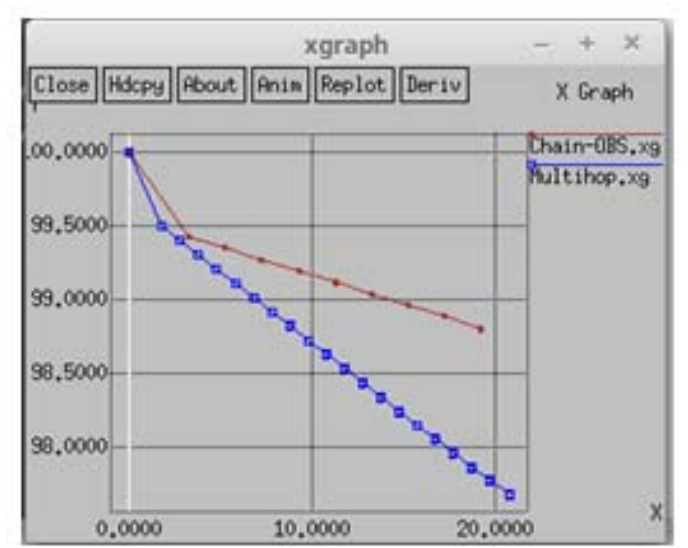

a) Multi hop [15] and multi hop Chain

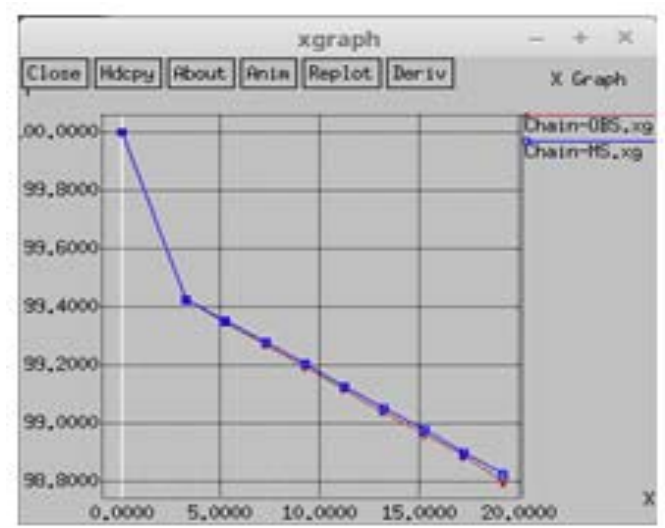

b) Multi hop Chain and Proposed

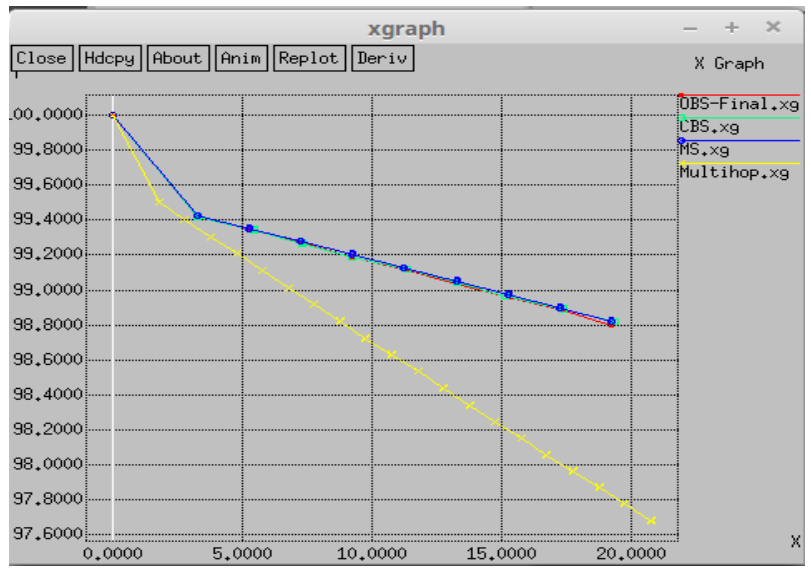

c) Over all comparison

Figure 6 Residual Energy comparisons.

Residual Energy: The remaining energy at the end of 20t, (simulation time) is as tabulated in table 2. Multi hop without chain [15] and chained OBS, in both sink is placed outside and results are Compared for the remaining energy at the end of $\mathrm{t}=20$, Figure $3 \mathrm{a}$. Un chained clusters with Multi hop intercommunication residual energy is around $97.5 \%$. Number ofmulti hop communications is equal to number of clusters formed in every’t'. More number of $\mathrm{CH}$ communicating to sink consumes more energy. Whereas when $\mathrm{CHs}$ are linked and only one chain leader i.e C-CH is allowed to communicate with sink, residual energy $98.80 \%$.For 20 t chaining process saves around $1.3 \%$ of energy.

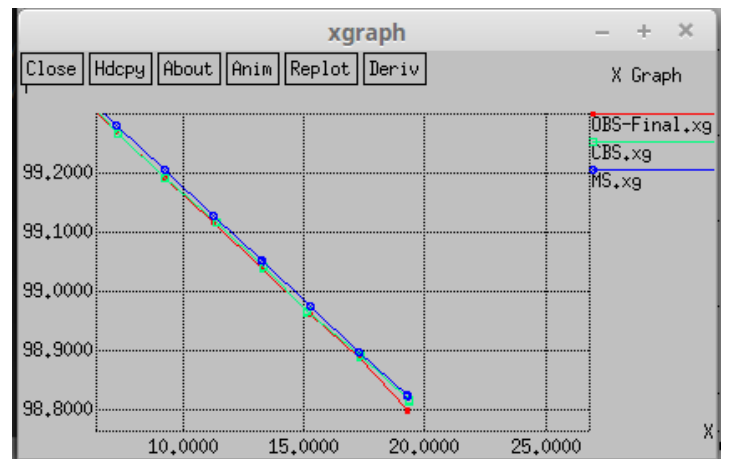

Figure 7. Effect of sink placement

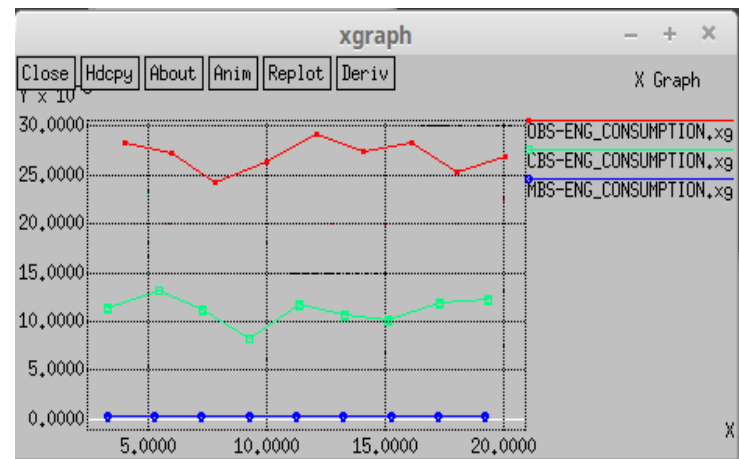

Figure 8. Energy Consumption

For the purpose of comparison OBS, CBS and MS use same clustering and chaining process they differ only in communication from C-CH to sink. The multi hop path length in OBS is more as every $\mathrm{CH}$ transmits its data to sink independently. Hence consume more energy. Therefore, over all networks' residual energy ofmulti hop without chain [15], is least when compared to other three techniques-OBS, CBS and MBS, Table 2, and Fig 3.c.

In Fig 4. The performance of OBS, CBS and MS are almost equal to one another in terms of residual energy. Initial MS position at the centre of sensor field yields some marginal benefits. 


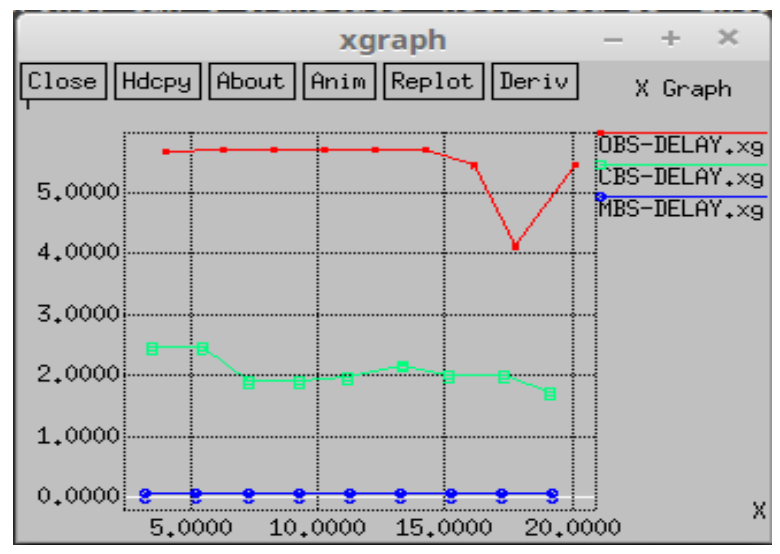

Figure 9. Delay comparison.

Energy consumed: Clustering and chaining procedure followed is same for OBS, CBS and MS. Hence energy consumption for communication from $\mathrm{CH}$ to $\mathrm{C}-\mathrm{CH}$ and then to sink is considered for comparison from table 3 , Chained OBS multi hop communication distance is reduced as accumulated data of all linked clusters is transmitted at once from chain head. Therefore, energy consumed in this case is less than multi hop without chain [15].

When sink is placed inside sensor field the path length is reduced. Resulting in short multi hop communication from C-CHs to CBS. Hence residual energy in network is more due to reduced energy consumption. MBS collects the data from chain head directly hence consumes less energy than CBS, Table 3, Figure 5. Energy consumption of proposed work is least. The residual energy with mobile sink with centre initial position is better than the other two cases, Figure 3.c.

Delay: In communication phase first, sensed data is collected at respective $\mathrm{CHs}$, further $\mathrm{CHs}$ forward their data to chain- $\mathrm{CH}$ node. As data collection is performed at two different levels data accumulation time increases. Chain process consumes more than conventional standalone cluster method. When sink is static and placed outside path length is more hence packets take more time to get delivered to sink. Due to nodes continuous movement their position changes and distance to sink is also varying. Therefore, total path length varies for every ' $t$ ', resulting an average delay of 4.913325t, forrandom 20t. Whereas static sink with initial position at centre results in reduced path length, hence average delay is less by $3.038495 \mathrm{t}$ for random $20 \mathrm{t}$.

In case of proposed work with MS the delay is constant as it collects data of all connected CHs from their C-CH. Hence data travels for zero distance from C-CH resulting in least and constant delay. The speed of MS is not considered for analysis and the delay is only of chain process.

From Table 4, MS has minimum latency when compared to other two sink cases. Time taken for Data transmission from C-CH to sink and energy consumption for these communications are proportional to reduced path length. From table 3, it is observed that as path length decreases energy consumed and data communication latency also decreases. The MS data collection by visiting the chain head nodes exhibit constant delay, Figure 6, and table 4.

TABLE 3: Performance Analysis

\begin{tabular}{|c|c|c|c|}
\hline & Static-OBS & Static-CBS & MBS \\
\hline Energy Consumed & $\begin{array}{c}2.43257 \mathrm{e}-05 \text { to } \\
2.91609 \mathrm{e}-05\end{array}$ & $\begin{array}{c}\mathbf{8 . 2 9 9 7 2 \mathrm { e } - 0 6} \text { to } \\
1.32443 \mathrm{e}-05\end{array}$ & $\begin{array}{c}3.53553 \mathrm{e}-07 \\
\text { (constant) }\end{array}$ \\
\hline Data collection Delay for 20t & 4.13411 to 5.6887 & 1.72855 to2.46982 & $\mathbf{0 . 0 7 0 7 1 0 7}$ (constant) \\
\hline Average delay & 4.913325 & 1.87483 & $\mathbf{0 . 0 7 0 7 1 0 7}$ Constant \\
\hline
\end{tabular}

\section{CONCLUSION:}

This work is a combination of multiple chains of linked CHs and MS forcollecting data from the chain heads. Independently these techniques have their own benefits and drawbacks. Linked clusters consume less energy than multi hop communication,but increases data delivery latency.MS reduces data latency, hence this work has reduced energy consumption and data collection latency and improves overall network residual energy.Results indicate chaining process is better than direct and multi hop routing.Hence the proposed chained CHs with MS for data collection work optimize overall network energy with reduced data delivery delay.

The simulationresults can be summarized as overall residual energy of traditionalmultihop routing is less than chained CHs. Sink placement at the centre of sensor field results in improved over all network residual energy when compared to outside sink. The data communication latency introduced by chaining process is reduced by 
MS. The delay comparison indicatesmobile sink collects data from minimum collection points with least communication delay when compared to static OBS and CBS.In future performance can be evaluated by using more than one MS. The effect of sensor range and dataaggregation to be studied.

\section{REFERENCE}

[1] S. Lindsey, C. Raghavendra, and K. M. Sivalingam, "Data GatheringAlgorithms in Sensor Networks using Energy Metrics",IEEETranscationon Parallel and Distributed System,2002, vol. 13, no. 9, pp. 924-935.

[2] Azharuddin, Md, PratyayKuila and Prasanta K. Jana,"Energyefficientfault tolerant clustering and routing algorithms for wireless sensornetworks",Computers and Electrical Engineering,2015, vol. no. 41, pp. 177-190.

[3] Abdul Waheed Khan, Abdul HananAbdullah, Mohammad HosseinAnisi, JavedIqbalBangash,"A Comprehensive Study of Data Collection Schemes Using Mobile Sinks in Wireless Sensor Networks",Sensors ,2014,14(2),pp 2510-2548.

[4] T. VenuMadhav and N V S N Sarma, "Energy Efficient Routing Protocolwith Improved Clustering Strategies for Homogeneous Wireless SensorNetworks",International Journal of Computer Applications, 2012,vol. 38, no.8, pp. 0975-8887.

[5] V. Raghunathan et al., "Energy-Aware Wireless MicrosensorNetworks",IEEE Signal Processing Magazine, 2002,vol. 1, no. 2, pp. 4050.

[6] W.R. Heinzelman, A. Chandrakasan, H. Balakrishnan, "Energy-efficientCommunication Protocol for Wireless Microsensornetworks",Hawaii International Conference on System Sciences(HICSS),vol 2, 2000,USA.

[7] S. Lindsey, C. S. Raghavendra, "PEGASIS: Power Efficient Gatheringin Sensor Information Systems",Proceedings of IEEE Aerospace Conference,March 2002.

[8] [8] Samaleswari P. Nayak, S. C. Rai, Sateesh K. Pradhan,"MERA-A Multi-Clustered Energy Efficient Routing Algorithm in WSN", International Conference on Information Technology,2015, pp 37-42.

[9] Feilong Tang,IlsunYou, SongGuo, MinyiGuo, YonggongMa"A chain-cluster based routing algorithm for wireless sensor networks", Journal of Intelligent Manufacturing, 2012, Vol 23, Issue 4, pp 1305-1313.

[10] Dawei Pan, Yu Peng, XiyuanPeng, "An energy-efficient link clustering algorithm for wireless sensor networks",IEEE International Conference on Electronic Measurement \& Instruments,2009,China.

[11] Mariam Alnuaimi ,KhaledShuaib , KlaithemAlnuaimi and Mohammed Abdel-Hafez, "Data Gathering in Delay Tolerant Wireless Sensor Networks Using a Ferry",Sensors,2015,15(10),pp 25809-25830

[12] Ming Ma,Yuanyuan Yang, Miao Zhao"Tour Planning for Mobile Data-Gathering Mechanisms in Wireless Sensor Networks",IEEE Transactions on Vehicular Technology, 2013, Vol 62, Issue: 4,pp 1427-1483.

[13] Miao Zhao, YuanyuanYang,Cong Wang "Mobile Data Gathering with Load Balanced Clustering and Dual Data Uploading in Wireless Sensor Networks", IEEE TRANSACTIONS ON MOBILE COMPUTING,2015, VOL. 14, NO. 4,pp 770-.785.

[14] KhaledAlmi’ani, AnastasiosViglas, LavyLibman," Energy-Efficient Data Gathering with Tour Length-Constrained Mobile Elements in Wireless Sensor Networks", IEEE Conference on Local Computer Networks , 2010,USA.

[15] Suma G, M Siddappa,"Life time improvement with hybrid clustering in Mobile Sensor Networks", International Journal of Computer Sciences and Engineering ,2017,vol 5,issue 11,pp 57-63.

\section{AUTHOR PROFILE}

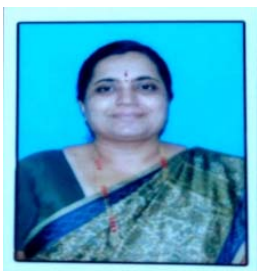

Mrs. Suma G has obtained her Bachelor's degree in Engineering from Bangalore university in Electronics and communication Engineering and her Master's degree in technology from Mysore university with a specialization in Industrial Electronics. She is pursuing her Doctoral degree from Sri Siddhartha Academy of Higher Education,Tumakuru. Her field of interest includes; wireless communication, GSM, CDMA, wireless sensor networks, mobile wireless sensor networks etc. Currently she is working as Associate professor in the Dept of Telecommunication Engineering, Sri Siddhartha Institute of Technology, Tumakuru. She has over 10 publications to her credit in various national/international journal/conferences of high repute. She has been the permanent member of Indian Society of Technical Institution and has guided more than 25 UG level final year dissertations.

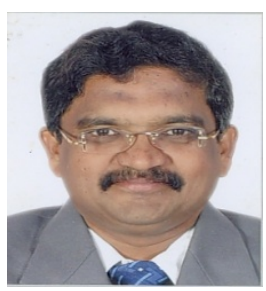

Dr M.Siddappa received B.E and M.Tech degree in Computer Science \& Engineering from University of Mysore, Karnataka, India in 1989 and 1993 respectively. He has completed doctoral degree from Dr.MGR Educational Research Institute Chennai under supervision of Dr.A.S.Manjunatha, CEO, Manvish e-Tech Pvt. Ltd., Bangalore in 2010. He worked as project associate in IISc, Bangalore under Dr.M. PSrinivasanand Dr.V.Rajaraman from 1993 - 1995. He has teaching experience of 29 years and research of 10 years. He published 72 Technical Papers in National, International Conference and Journals. He has citation index of 236 till 2016 and h-index of 7 and i10-index of 5 to his credit. He is a member of IEEE and Life member of ISTE. He is working in the field of data structure and algorithms, Artificial Intelligence, Image processing and Computer networking. He worked as Assistant Professor in Department of Computer Science \& Engineering from 1996 to 2003 in Sri Siddhartha Institute of Technology, Tumkur. Presently, he is working as Professor and Head, Department of Computer Science \& Engineering from 1999 in Sri Siddhartha Institute of Technology, Tumkur. He has visited Louisiana university Baton rouge, California university and Wuhan university China. He has been awarded Best Teacher-ISTE 2013. 